\title{
Spontaneous rupture of the dissected coronary sinus mimicking acute coronary syndrome
}

Thomas Bartel, MD, ${ }^{\mathrm{a}}$ Alfred Kocher, MD, ${ }^{\mathrm{b}}$ Gudrun Feuchtner, MD, ${ }^{\mathrm{c}}$ Silvana Müller, MD, ${ }^{\mathrm{a}}$ Johannes O. Bonatti, MD, ${ }^{\mathrm{c}}$ and

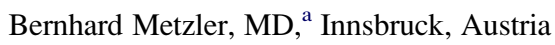

Video clip is available online.

Coronary sinus (CS) dissection has been described as a complication of pacing electrode implantation only. ${ }^{1}$ We report here the diagnosis and surgical therapy of spontaneous rupture of a dissected $\mathrm{CS}$ in a patient with acute coronary syndrome (ACS) and circumflex artery (CX) to the CS fistula.

\section{CLINICAL SUMMARY}

ACS was suspected in a 63-year-old man with acute chest pain after physical exertion but with no electrocardiographic signs of acute myocardial infarction. Coronary angiography showed a giant left main coronary artery aneurysm. A very ectatic and calcified CX originated from the aneurysm and drained into a huge CS. There were no coronary stenoses. Transesophageal echocardiographic analysis revealed a possibly hemorrhagic pericardial effusion. Septated echodense structures were found within the CS lumen that were indicative of chronic dissection (Figure 1, $A$ ). Turbulent flow inside the CS was indicative of arterial pressure (Figure 1,B). Cardiac electrocardiographically gated 64-slice computed tomography confirmed a fistula ${ }^{2}$ passing along the left atrioventricular groove, taking a tortuous course with multiple loops before draining into the CS (Figure 1, $C$ and $D$ ). The posterolateral and great cardiac veins were also markedly dilated because of chronic pressure overload (Figure 1, $E$ and $F$ ). Consequently, a concealed perforation was thought to have caused hemorrhage. At the time of emergency surgical intervention, approximately $300 \mathrm{~mL}$ of hemorrhagic pericardial effusion and blood clots was removed. Manipulation of the multisegmented and enlarged CS was avoided. The

\footnotetext{
From the Cardiology Division, Department of Internal Medicine, ${ }^{a}$ the Department of Cardiac Surgery, ${ }^{\mathrm{b}}$ and Institute of Radiology, ${ }^{\mathrm{c}}$ Medical University Innsbruck, Innsbruck, Austria.

Received for publication Feb 6, 2008; revisions received Feb 6, 2008; accepted for publication Feb 17, 2008.

Address for reprints: Thomas Bartel, MD, Cardiology Division, Department of Internal Medicine, University of Innsbruck, Anichstr. 35, A-6020 Innsbruck, Austria (E-mail: thomas.bartel@i-med.ac.at).

J Thorac Cardiovasc Surg 2009;137:e1-3

$0022-5223 / \$ 36.00$

Copyright (c) 2009 by The American Association for Thoracic Surgery

doi:10.1016/j.jtcvs.2008.02.036
}

fistula was closed, the main stem and CX were dissected, and a large part of the diffuse aneurysm was excised (Figure 2), taking care to leave enough tissue behind next to the orifice of the left anterior descending coronary artery and first diagonal branch, which both originated from the aneurysm back to back. A roof was constructed, with part of the aneurysm wall reinforced by pericardium patches to ensure communication between the left anterior descending coronary artery and the intermediate branch. Subsequently, coronary revascularization was successfully completed, with the left internal thoracic artery bypassing the left anterior descending coronary artery and a saphenous vein graft to the largest obtuse-marginal branch. The patient recovered from surgical intervention uneventfully and was discharged home on postoperative day 6 .

\section{DISCUSSION}

To our knowledge, this case demonstrates for the first time that (1) spontaneous dissection of the CS can occur because of pressure overload as a result of a CX-to-CS fistula and can be adequately visualized by means of transesophageal echocardiography $^{3}$; (2) CS dissection can be complicated by pericardial hemorrhage; and (3) CS dissection can mimic not only coronary artery disease by a "steal" phenomenon ${ }^{3}$ but also ACS caused by painful perforation and should therefore be considered in the differential diagnosis of patients with acute chest pain and pericardial effusion.

\section{References}

1. Yoda M, Hansky B, Koerfer R, Minami K. Coronary sinus dissection during left ventricular pacing electrode implantation. Ann Thorac Cardiovasc Surg. 2007; 13:275-7.

2. De Cicco G, Fucci C, Borghesi A, Lorusso R. Left circumflex coronary artery to coronary sinus fistula. Eur J Cardiothorac Surg. 2006;30:559.

3. Bothe W, Schlensak C, van de Loo A, Beyersdorf F. A fistula between the circumflex artery and the coronary sinus mimics coronary artery disease in a 63 -year-old woman. Interact Cardiovasc Thorac Surg. 2005;4:81-2. 

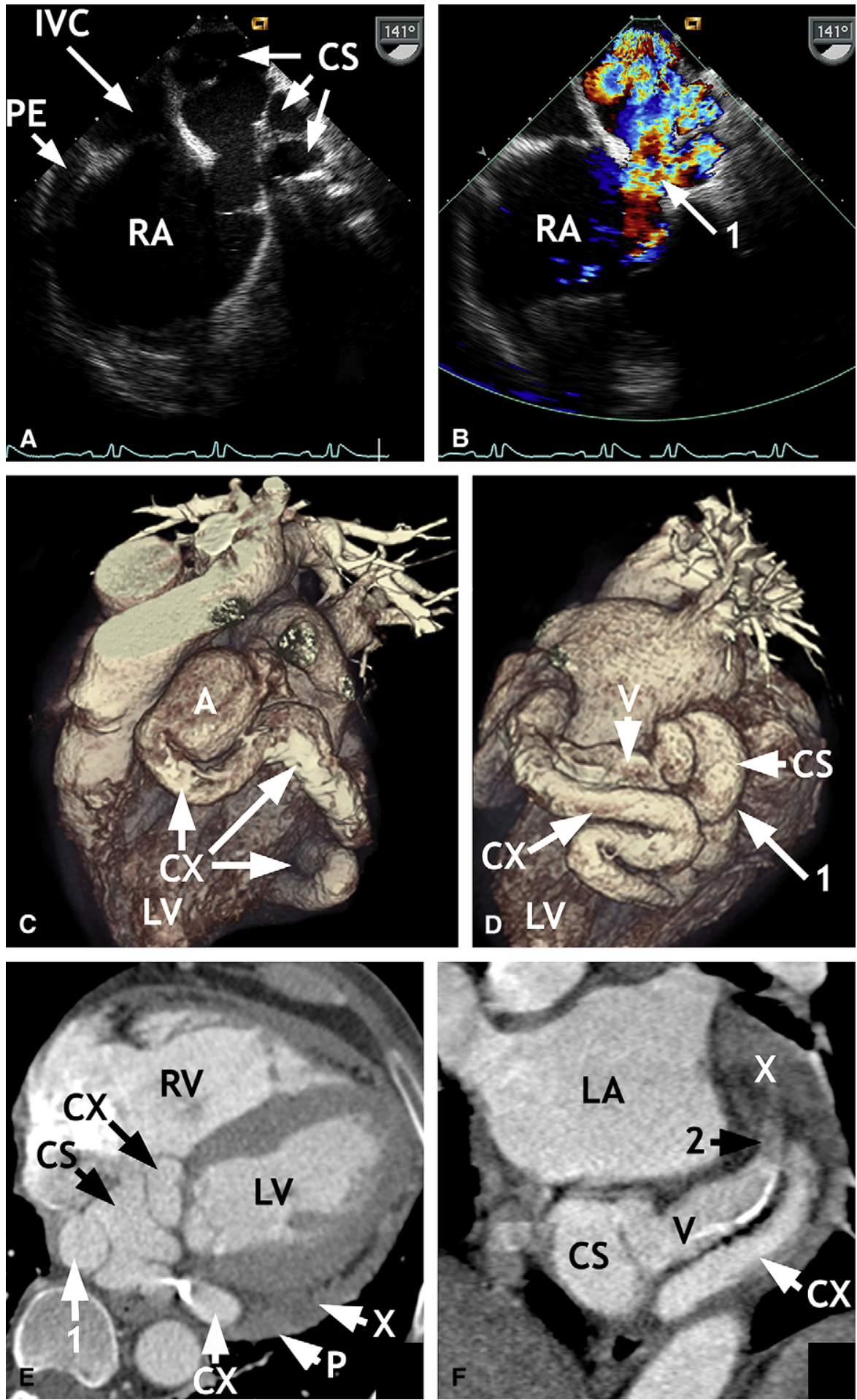

FIGURE 1. A and B, Enlarged and dissected coronary sinus (A) with turbulent fistular flow (B). 1 , Turbulent entrance flow from the coronary sinus into the right atrium; $C S$, coronary sinus; $I V C$, inferior vena cava; $P E$, pericardial effusion; $R A$, right atrium. $\mathrm{C}$ and $\mathrm{D}$, Computed tomographic 3-dimensional view of the heart by using a volume-rendering technique: giant left main coronary aneurysm (anterior view of the heart; C) from which an enlarged, heavily calcified circumflex artery originates, which drains distally into the dilated, septated coronary sinus (posterior view of the heart; D). A, Aneurysm; CS, coronary sinus; $C X$, circumflex coronary artery; $L V$, left ventricle; $V$, dilated posterolateral cardiac vein; 1 , the fistula between circumflex coronary artery and coronary sinus. $\mathrm{E}$ and F, Computed tomographic axial oblique (E) and coronal oblique $(\mathrm{F})$ planes of the heart. The circumflex artery drains into the dissected coronary sinus (E). The distal posterolateral vein is enlarged and shows thrombus formation and surrounding hemorrhage indicative of a concealed perforation (F). $L V$, left ventricle; $C S$, coronary sinus; $C X$, circumflex coronary artery; $V$, distal posterolateral vein; $P$, pericardium; $X$, hemorrhage; $L A$, left atrium; $R V$, right ventricle; 1 , the circumflex artery drains into the dissected coronary sinus; 2 , thrombus formation in the posterolateral vein. 

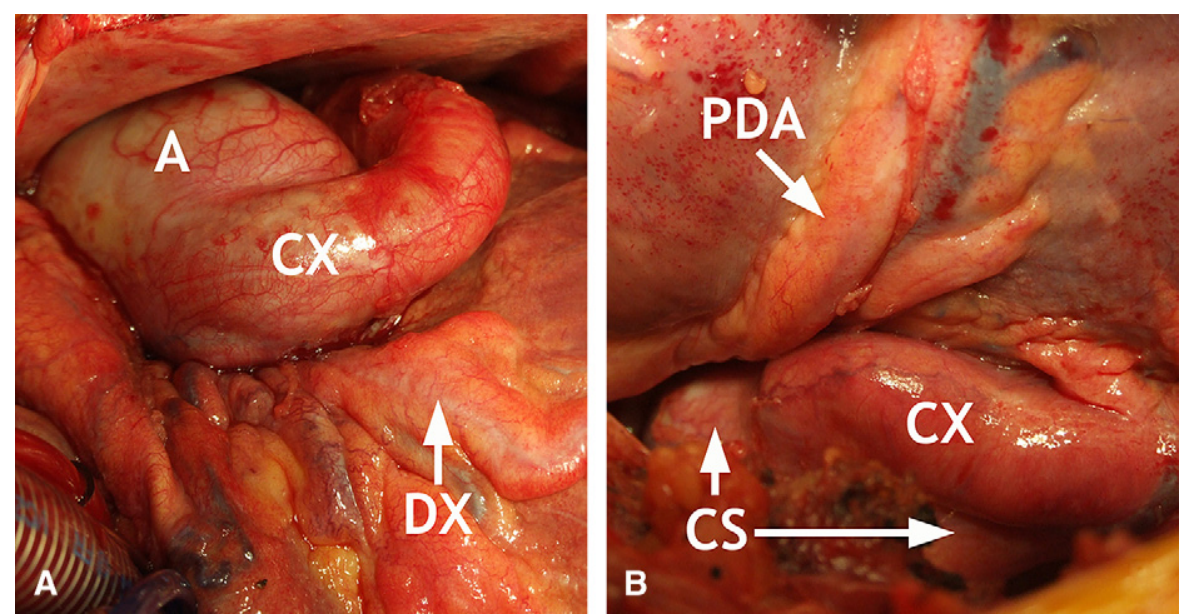

FIGURE 2. Intraoperative view of the junction between the left main coronary aneurysm and the ectatic circumflex artery (A) and the circumflex artery to the coronary sinus fistula (B). A, Left main aneurysm; $C S$, coronary sinus; $C X$, circumflex artery; $D X$, first diagonal coronary artery; $P D A$, posterior descending coronary artery.

\section{Lipomatous septal hypertrophy}

Christina Doesch, MD, Claudia Plachtzik, MD, and Christian S. Haas, MD, Tuebingen, Germany

A 76-year old obese woman with a body mass index (BMI) of 36.8 was referred for evaluation of an intracardiac mass, incidentally detected with computed tomography (CT). The CT scan showed massive thickening of the intra-atrial septum with the septal tissue clearly hypointense to normal myocardium and isointense to subcutaneous fat (Figure 1, $A$ ) and sparing of the fossa ovalis (Figure 1, B, arrows). Cardiac magnetic resonance imaging confirmed septal thickening of either side of the foramen ovale (Figure 1, C, arrows), being isointense to the subcutaneous fat. Echocardiographic studies (Figure 1,D) revealed hypertrophy of the atrial septum with bright echogenicity of the thickened tissue and

\footnotetext{
From the University Hospital Tuebingen, Department of Internal Medicine, Division of Cardiology and Cardiovascular Medicine, Tuebingen, Germany.

Received for publication Jan 2, 2008; accepted for publication Jan 12, 2008.

Address for reprints: Christian S. Haas, MD, University Hospital Tuebingen, Department of Internal Medicine, Division of Cardiology and Cardiovascular Medicine, Otfried-Mueller-Strasse 10, 72076 Tuebingen, Germany (E-mail: cs_haas@ yahoo.com).

J Thorac Cardiovasc Surg 2009;137:e3-4

$0022-5223 / \$ 36.00$

Copyright (c) 2009 by The American Association for Thoracic Surgery

doi:10.1016/j.jtcvs.2008.01.034
}

a typical dumbbell-shaped appearance. The diagnosis of lipomatous hypertrophy of the interatrial septum was made.

Lipomatous septal hypertrophy is a rare, benign, often asymtomatic condition primarily seen in older patients and is frequently confused with other cardiac tumors. ${ }^{1,2}$ Histologically, it is characterized by proliferation of adipocytes and interspersed hypertrophied cardiomyocytes. ${ }^{3}$ With imaging studies, lipomatous septal hypertrophy is indistinguishable from lipomas but occurs exclusively in the atrial septum and has no tendency to grow like a tumor. Massive fat deposition in the atrial wall results in a thickness of the atrial septum greater than $20 \mathrm{~mm}$ up to $60 \mathrm{~mm}^{3}$ and is associated with a higher incidence of atrial arrhythmias. ${ }^{1}$ The diagnosis of lipomatous septal hypertrophy can be established by complementary imaging studies using transthoracic or transesophageal echocardiography, CT scanning, and magnetic resonance tomography. Characteristic features include a typical single mass in the interatrial septum, which is isointense to subcutaneous fat and does not involve the fossa ovalis. ${ }^{1,4}$ In the absence of severe arrhythmias, conduction problems, or signs of obstruction owing to protrusion into the atrium, no specific therapy is required. ${ }^{2,3,5}$ 